\title{
Die Baumbedeckung in der Schweiz
}

\author{
Christian Ginzler \\ Lukas Mathys \\ Esther Thürig
}

\author{
Eidgenössische Forschungsanstalt für Wald, Schnee und Landschaft $(\mathrm{CH})^{*}$ \\ Sigmaplan AG $(\mathrm{CH})$ \\ Eidgenössische Forschungsanstalt für Wald, Schnee und Landschaft $(\mathrm{CH})$
}

\begin{abstract}
Tree cover in Switzerland
The requirements for national forest inventories have changed in recent decades, as have the issues involved. Initially, the focus was mainly information on timber resources, but today social and environmental functions are also of interest. An a priori separation of the surveyed areas into forest and non-forest during data collection limits the interpretation of the tree resources. Not all trees are located in the forest and not all forests are fully stocked. In the aerial photo interpretation of the $3^{\text {rd }}$ National Forest Inventory, land cover on a regular sampling grid was determined regardless of the land use. This allowed, for the first time, nationwide information on tree resources to be obtained, independent of forest definitions. The tree cover of Switzerland is $27.0 \%$ regardless of whether the trees are in the forest or outside. The area covered with forest (proportionally 29.4\%) is larger than that covered with trees. The location of the trees outside the forest tends to be mostly either very close to forest or in urban areas. The most densely stocked areas, after forests, are urban areas. The data from aerial photo interpretations of the $3^{\text {rd }}$ National Forest Inventory allow a more nuanced picture of the stocking over the whole country, but the sampling error is still too large to draw conclusions for small areas. The existing and ongoing surveys, however, provide a calibration and reference dataset so that, with remote sensing data and methods, it should be possible to generate more comprehensive spatial datasets to help to fill this gap.
\end{abstract}

Keywords: forest inventory, landscape analysis, tree inventory, trees outside forest, aerial photo interpretation doi: 10.3188.szf.2011.0344

* Zürcherstrasse 111, CH-8903 Birmensdorf, E-Mail christian.ginzler@wsl.ch
$\mathrm{D}$ ie Erfassung der Schweizer Holzressourcen hat eine lange Tradition. Ursprünglich interessierte hauptsächlich die Erfassung ökonomisch relevanter Grössen wie des Holzvorrats (Kimmins 2004). Dementsprechend beschränkte sich die Wahrnehmung und Messung von Bäumen auf Flächen innerhalb des Waldes. Alles ausserhalb des Waldes war zu Beginn der meisten Inventuren ohne Belang. Ab der zweiten Hälfte des 20. Jahrhunderts erweiterten sich die Ansprüche an die Holzressourcen (Brassel et al 1999). Neben der Wirtschaftsfunktion kamen gesellschaftliche und ökologische Funktionen wie Erholung, Lebensraum für Organismen oder Speicher von Kohlenstoff dazu (Millennium Ecosystem Assessment 2005).

Bei langfristig angelegten Inventuren können sich also im Laufe der Zeit das Ziel und der Zweck der Inventur wandeln. Steht wie früher hauptsächlich die Frage des Holzvorrats im Zentrum, reicht ein einfaches, spezifisches Inventurdesign mit wenigen ausgewählten Methoden. Je breiter jedoch die zu quantifizierende Funktionspalette und je unbekann- ter die zukünftigen Fragestellungen sind, desto anspruchsvoller sind das Design und die Durchführung von Inventuren (Lanz \& Ginzler 2005). Bei einer langfristigen Inventur sollten die Daten somit möglichst allgemeingültig erhoben werden, das heisst ohne einschränkende Vorauswahl. Beim dritten Schweizerischen Landesforstinventar (LFI3) wurde diesem Umstand insofern Rechnung getragen, als in die Luftbildauswertung alle Bäume der Schweiz einbezogen wurden. Im LFI3 wurden dementsprechend die für den Wald-Nichtwald-Entscheid notwendigen Rohdaten aus dem Luftbild schweizweit auf allen Probeflächen im regelmässigen Abstand von 500 m erfasst. Daraus entstand ein Datensatz, mit dem unabhängig von einer Walddefinition die Bodenbedeckung und damit auch die Bäume erfasst wurden. Durch die strikte Trennung von Bodenbedeckung und Landnutzung konnten erstmals schweizweit repräsentative und quantitative Informationen zu Bäumen unabhängig von vordefinierten Landnutzungskategorien gewonnen werden. Der erste Schritt von einer Waldinventur hin zu einer 
Landschaftsinventur wurde damit gemacht (Mathys et al 2005).

Ziel der vorliegenden Untersuchung war, die Verteilung der Wald- und Nichtwaldbäume in der Schweiz auf der landschaftlichen Ebene zu quantifizieren und deren Umgebung durch andere Bodenbedeckungen auf der lokalen Ebene zu analysieren.

\section{Methode}

\section{Datenerhebung}

Die Rohdaten wurden im LFI3 schweizweit auf einem regelmässigen Stichprobenetz mit $500 \times 500 \mathrm{~m}$ Abstand erhoben (Brassel \& Lischke 1999). Dies ergibt 165153 Stichproben. Jede einzelne Stichprobe bestand aus einer quadratischen Probefläche mit $50 \mathrm{~m} \times 50 \mathrm{~m}$ Ausdehnung. Auf diesen Probeflächen wurden jeweils $5 \times 5$ Rasterpunkte im Abstand von 10 m erhoben (Abbildung 1). Diese manuelle Datenerhebung auf Echtfarbenstereobildern mit einer Bodenauflösung von $0.5 \mathrm{~m}$ erfolgte in zwei Schritten.

In einem ersten Schritt wurden die $5 \times 5$ Rasterpunkte pro Probefläche einer von elf Bodenbedeckungskategorien zugeordnet (Ginzler et al 2005). Die möglichen Kategorien waren Laubbaum, Nadelbaum, Lärche, Strauch, Kraut-/Grasvegetation, Felsen, Sand/Gestein/Offenerde, befestigte Fläche, Bauobjekt, Gewässer und Gletscher/Firn. Die Zuordnung der 25 Rasterpunkte erfolgte völlig unabhängig davon, ob eine Probefläche zum Wald gehörte oder nicht. Ein Rasterpunkt auf einem zum Wald gehörenden Baum wurde gleich interpretiert wie ein Rasterpunkt auf einem städtischen Parkbaum oder einem landwirtschaftlich genutzten Obstbaum. Die Luftbildinterpretation konzentrierte sich somit im ersten Schritt ausschliesslich auf die Bodenbedeckung, unabhängig von der Nutzungskategorie wie zum Beispiel Wald oder Nichtwald. Wo die Raster-

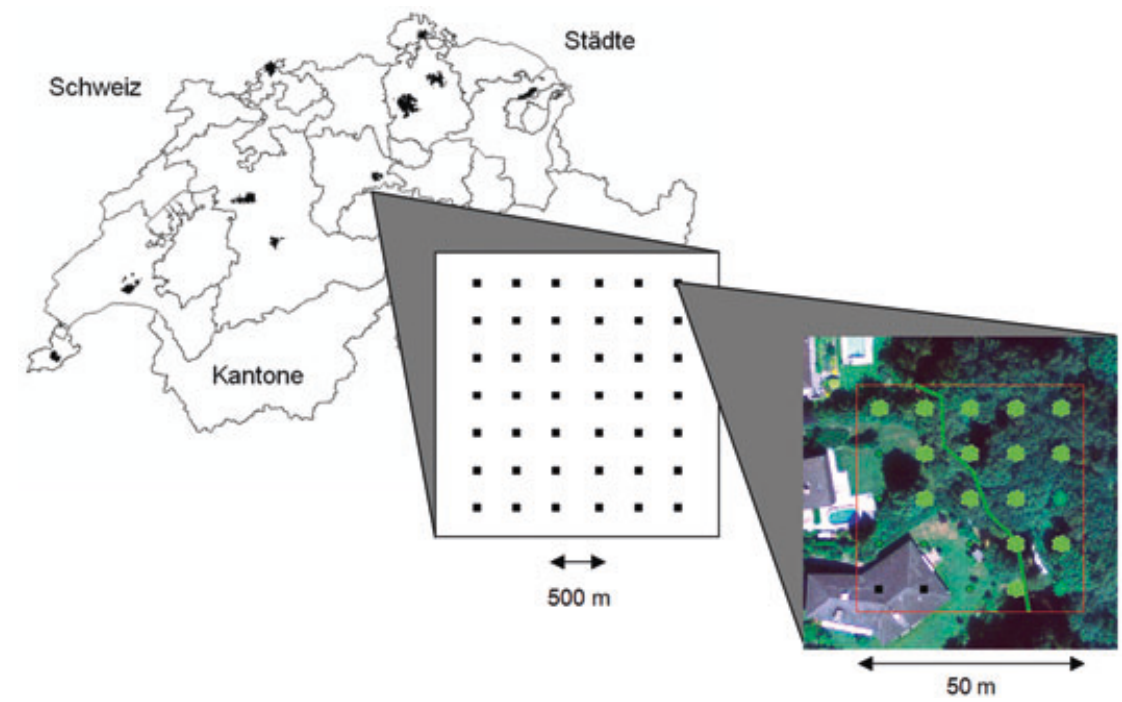

Abb 1 Regelmässiges Stichprobenetz mit regelmässig angeordneten Rasterpunkten. Auswertungseinheiten sind die Schweiz, die Kantone und die zehn grössten Städte. punkte die Oberfläche berührten, wurde zudem die Höhe über Meer gemessen, die entsprechende Geländehöhe aus dem digitalen Terrainmodell der amtlichen Vermessung mit einer Rasterweite von $2 \mathrm{~m} \times 2 \mathrm{~m}$ (DTM-AV, (C) 2010 Swisstopo) zugewiesen und daraus die Höhe des entsprechenden Elementes abgeleitet. Wurde die Interpretationsfläche von einem Waldrand geschnitten, so wurde zusätzlich eine Waldbegrenzungslinie kartiert.

Im zweiten Schritt wurden für jede Probefläche $(50 \mathrm{~m} \times 50 \mathrm{~m})$ anhand der Rasterpunkte und der kartierten Waldbegrenzungslinie Flächeneigenschaften wie Deckungsgrad, Breite und Oberhöhe abgeleitet und darauf basierend der Wald-Nichtwald-Entscheid gefällt (Ginzler et al 2005). Damit wurde auch bestimmt, welche Probeflächen in der anschliessenden terrestrischen Waldinventur aufgesucht werden und welche nicht.

Bäume innerhalb und ausserhalb des Waldes

Die Klassierung der Bäume in Wald- und Nichtwaldbäume für diese Untersuchung erfolgte anhand 1) des Waldentscheides für die Probefläche, 2) der Bodenbedeckung und 3) der Elementhöhe der Rasterpunkte sowie 4) der Lage in einer Waldecke im Falle einer Nichtwaldprobe. Um also einen Rasterpunkt als Nichtwaldbaum zu klassieren, musste die Probefläche Nichtwald, die Bodenbedeckung Nadelbaum, Laubbaum oder Lärche, die Elementhöhe des Rasterpunktes über dem Boden mindestens $3 \mathrm{~m}$ und die Lage nicht in einer Waldecke sein. Liegt die Probefläche im Wald, werden alle anderen Rasterpunkte mit obigen Bodenbedeckungen und einer Höhe von mindestens $3 \mathrm{~m}$ dem Wald zugeordnet.

\section{Auswertungseinheiten}

Die Quantifizierung der räumlichen Baumverteilung erfolgte schweizweit, nach Kantonen und den zehn siedlungsanteilsmässig grössten Städten. Dafür wurden die politischen Gemeindegrenzen (BFS Geostat) mit den Siedlungsgrenzen des Vector25-Datensatzes (Swisstopo) verschnitten. Die Daten wurden nach Landnutzungskategorien, wie den beschriebenen Wald- und Nichtwaldarealen, sowie den baumorientierten Nutzungsarealen (Tabelle 1) ausgewertet. Diese Nutzungsareale wurden aus dem vorhandenen Landschaftsmodell Vector25 (ㄷ) 2010 Swisstopo) abgeleitet. Um die Datenlayer des Landschaftsmodells wurden abhängig von der Landnutzung Puffer mit unterschiedlicher Breite (Tabelle 1) gelegt. Jeder Rasterpunkt wurde mit allen elf thematischen Nutzungsarealen verschnitten. Weil sich die Kategorien überlappen können, wurde eine Priorisierung vorgenommen (Tabelle 1, rechte Spalte). Ist zum Beispiel ein Punkt siedlungsnah und bachnah, so hat bachnah eine höhere Priorität. Wurde ein Punkt von keinem Nutzungsareal geschnitten, so wurde er dem Areal Landwirtschaft zugewiesen. 


\begin{tabular}{|c|c|c|c|}
\hline Nutzungsareal & $\begin{array}{l}\text { Pufferbreite } \\
\text { (m) }\end{array}$ & Kodierung im Vector25-Datenlayer & Priorität \\
\hline Landwirtschaft & - & $\begin{array}{l}\text { Alle Punkte, die nicht von einem } \\
\text { Datenlayer geschnitten werden }\end{array}$ & 1 \\
\hline Obstbau & 5 & Z_ObstAn & 2 \\
\hline Weinberge & 5 & Z_Reben & 3 \\
\hline Bäche & 5 & Bach, Kanal, Bisse & 4 \\
\hline Flüsse & 25 & Z_Fluss, Z_See & 5 \\
\hline Grosse Strassen & 15 & $\begin{array}{l}\text { AutoRte, Autob_Ri, Autobahn, } \\
\text { Autostr, AutrReA, AutrRtrB }\end{array}$ & 6 \\
\hline Siedlung & 5 & Z_Siedl & 7 \\
\hline Eisenbahn & 10 & $\begin{array}{l}<>\text { Str_Bahn, Str_Bhof, I_Geleis von } \\
\text { Railroad }\end{array}$ & 8 \\
\hline Kleine Strassen & 5 & 1_Klass, 2_Klass & 9 \\
\hline Gebäude & 25 & $\begin{array}{l}\text { Z_Gasthof, Z_Gebaeude, Z_Huette, } \\
\text { Z_Kirche }\end{array}$ & 10 \\
\hline Wald $20 \mathrm{~m}$ & 20 & $\begin{array}{l}\text { Z_GerWa, Z_SumWa, Z_Wald, } \\
\text { Z_WaldOf, Z_GerWaO, Z_Sum- } \\
\text { WaO, Z_SumGeb, Z_Gebue }\end{array}$ & 11 \\
\hline Wald $50 \mathrm{~m}$ & 50 & $\begin{array}{l}\text { Z_GerWa, Z_SumWa, Z_Wald, } \\
\text { Z_WaldOf, Z_GerWaO, Z_Sum- } \\
\text { WaO, Z_SumGeb, Z_Gebue }\end{array}$ & 12 \\
\hline
\end{tabular}

Tab 1 Baumorientierte Nutzungsareale abgeleitet aus den Objektklassen Vector25. Für die Kodierung des Vector25-Datenlayers siehe www.swisstopo.ch.

\begin{tabular}{|l|c|c|c|c|c|c|}
\hline Kanton & Waldanteil & $\begin{array}{c}\text { Standard- } \\
\text { fehler } \\
(\%)\end{array}$ & $\begin{array}{c}\text { Baum- } \\
\text { anteil } \\
(\%)\end{array}$ & $\begin{array}{c}\text { Standard- } \\
\text { fehler } \\
(\%)\end{array}$ & $\begin{array}{c}\text { Bäume im } \\
\text { Wald } \\
(\%)\end{array}$ & $\begin{array}{c}\text { Bäume im } \\
\text { Nichtwald } \\
(\%)\end{array}$ \\
\hline AG & 34.9 & 0.7 & 37.5 & 0.5 & 91.2 & 8.8 \\
\hline Al & 31.1 & 2.3 & 28.9 & 1.1 & 96.2 & 3.8 \\
\hline AR & 33.9 & 1.9 & 34.9 & 1.0 & 96.0 & 4.0 \\
\hline BE & 29.5 & 0.4 & 27.9 & 0.2 & 93.9 & 6.1 \\
\hline BS/BL & 44.2 & 1.4 & 45.3 & 0.7 & 90.9 & 9.1 \\
\hline FR & 26.0 & 0.7 & 25.0 & 0.4 & 91.7 & 8.3 \\
\hline GE & 12.1 & 1.1 & 20.0 & 0.8 & 60.9 & 39.1 \\
\hline GL & 25.8 & 1.3 & 22.1 & 0.6 & 96.9 & 3.1 \\
\hline GR & 24.9 & 0.3 & 18.5 & 0.2 & 97.8 & 2.2 \\
\hline JU & 40.1 & 1.0 & 42.4 & 0.6 & 95.2 & 4.8 \\
\hline LU & 27.9 & 0.7 & 28.8 & 0.4 & 92.0 & 8.0 \\
\hline NE & 36.6 & 1.1 & 35.6 & 0.6 & 94.9 & 5.1 \\
\hline NW & 29.8 & 1.8 & 27.9 & 1.0 & 95.6 & 4.4 \\
\hline OW & 40.7 & 1.4 & 32.8 & 0.7 & 97.0 & 3.0 \\
\hline SG & 28.2 & 0.7 & 28.1 & 0.3 & 92.6 & 7.4 \\
\hline SH & 41.7 & 1.7 & 44.1 & 1.0 & 95.1 & 4.9 \\
\hline SO & 41.4 & 1.0 & 42.9 & 0.6 & 93.6 & 6.4 \\
\hline SZ & 34.2 & 1.0 & 28.2 & 0.5 & 94.8 & 5.2 \\
\hline TG & 21.5 & 0.8 & 23.8 & 0.5 & 87.9 & 12.1 \\
\hline TI & 47.8 & 0.6 & 41.2 & 0.3 & 97.3 & 2.7 \\
\hline UR & 17.4 & 1.0 & 12.6 & 0.4 & 96.7 & 3.3 \\
\hline VD & 31.6 & 0.5 & 29.4 & 0.3 & 93.4 & 6.6 \\
\hline VS & 22.3 & 0.4 & 17.7 & 0.2 & 95.0 & 5.0 \\
\hline ZG & 25.1 & 1.7 & 27.4 & 1.0 & 89.0 & 11.0 \\
\hline ZH & 28.7 & 0.7 & 32.4 & 0.4 & 88.3 & 11.7 \\
\hline CH & 29.4 & 0.1 & 27.0 & 0.1 & 93.9 & 6.1 \\
\hline & & & & & & 5 \\
\hline
\end{tabular}

Tab 2 Verteilung der Bäume und des Waldes nach Kantonen und für die gesamte Schweiz.

\section{Ergebnisse und Diskussion}

\section{Baumverteilung in der Schweiz}

Der Baumanteil der Schweiz beträgt 27.0\%, unabhängig davon, ob die Bäume im Wald, in der Siedlung oder der übrigen Landschaft stehen (Tabelle 2). Diese Zahl berechnet sich aus der Summe aller Baumrasterpunkte (1114825) dividiert durch die gesamte Summe aller interpretierten Rasterpunkte (165153 × 25 Rasterpunkte). Der Waldflächenanteil der Schweiz beträgt $29.4 \%$ der Landesfläche. Die Waldfläche ist somit grösser als die mit Bäumen überschirmte Fläche. Dies lässt sich durch die Walddefinition des LFI erklären, wo schon ein Deckungsgrad von 20\% für einen positiven Waldentscheid genügt. Die Bäume liegen schweizweit zu 93.9\% innerhalb und zu 6.1\% ausserhalb des Waldes (Tabelle 2).

Der Wald- respektive Baumanteil ist regional sehr unterschiedlich (Tabelle 2). Den höchsten Waldanteil weist der Kanton Tessin mit 47.8\% auf, am wenigsten Wald hat der Kanton Genf mit 12.1\% der Fläche. Anteilsmässig die meisten Bäume hat der Kanton Basel (BS/BL, 45.3\%), am wenigsten der Kanton Uri (12.6\%). Das Verhältnis von Wald- zu Baumanteil scheint jedoch unabhängig davon gleichbleibend zu sein (Abbildung 2), weil alle Punkte mehr oder weniger auf der 1:1-Linie liegen und eine maximale Anteilsdifferenz von 7.9\% (Kantone Obwalden und Genf) aufweisen. Dies bedeutet, dass es für den Anteil der Bäume im Nichtwaldareal entsprechende Flächen ohne Bäume im Waldareal geben muss (Windwurfflächen, Baumlücken, Schlagflächen, Waldstrassen etc.). In rund zwei Dritteln der Kantone ist der Waldanteil grösser als der Baumanteil. Dies vor allem in Kantonen mit Flächen an der oberen Waldgrenze mit aufgelösten Beständen. In Anbetracht dessen, dass ursprünglich grosse Teile der Schweizer Landesfläche von Bäumen bedeckt waren, zeigen die Resultate aber auch, dass die Nichtwaldlandschaft bezüglich Bäumen ausgeräumt ist. Der regionale Vergleich zeigt zudem, dass hinsichtlich der Bäume das Nichtwaldareal in ländlichen Regionen stärker ausgeräumt ist als in städtischen. Dies zeigt sich speziell am Beispiel des Kantons Genf, wo der Baumanteil mit 20\% rund 8\% grösser ist als der Waldanteil (Abbildung 2, grösster Punkt).

\section{Baumverteilung ausserhalb des Waldes}

Der Anteil (Tabelle 2) und die räumliche Verteilung der Bäume ausserhalb des Waldes (Tabelle 3) unterscheiden sich regional stark. Im Folgenden wird die räumliche Verteilung genauer betrachtet.

Je ländlicher eine Region ist, desto weniger Bäume stehen anteilsmässig ausserhalb des Waldes. In einigen ländlicheren Regionen wie zum Beispiel den Kantonen Appenzell Innerrhoden oder Graubünden stehen diese Bäume vermehrt in Waldes- 


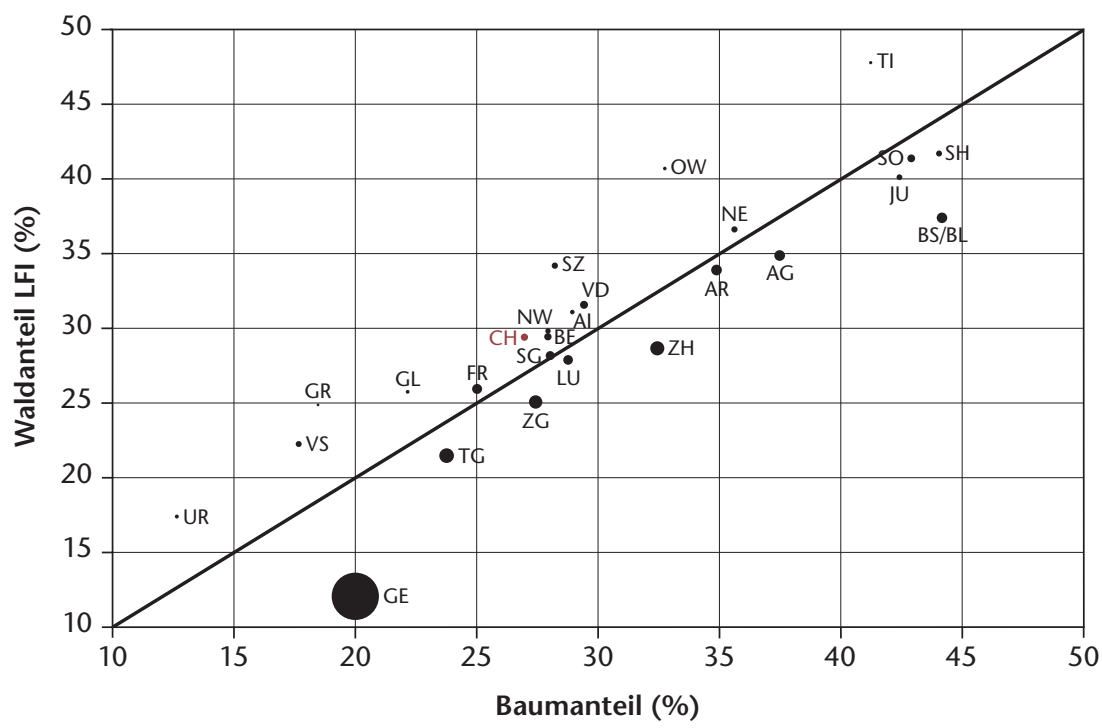

Abb 2 Wald- und Baumanteil in den Kantonen. Die Grösse der Kreise repräsentiert den Anteil der Bäume ausserhalb des Waldes. Je grösser der Kreis, desto höher der Anteil an Bäumen im Nichtwald.

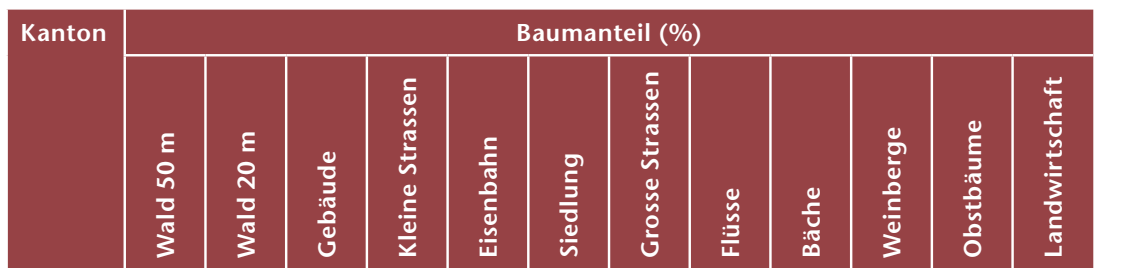

\begin{tabular}{|c|c|c|c|c|c|c|c|c|c|c|c|c|}
\hline $\mathrm{AG}$ & 4.7 & 11.7 & 5.6 & 1.1 & 0.1 & 39.9 & 0.9 & 7.6 & 4.8 & 0.3 & 6.0 & 17.2 \\
\hline $\mathrm{Al}$ & 7.3 & 49.9 & 8.2 & 0.2 & 0.0 & 7.1 & 0.0 & 4.5 & 8.9 & 0.0 & 0.5 & 13.4 \\
\hline AR & 8.8 & 34.3 & 15.2 & 0.9 & 0.0 & 17.3 & 0.5 & 2.4 & 4.5 & 0.0 & 2.9 & 13.1 \\
\hline$B E$ & 9.5 & 26.6 & 8.7 & 1.3 & 0.2 & 22.7 & 0.6 & 5.5 & 4.7 & 0.2 & 2.5 & 17.5 \\
\hline$B L$ & 3.7 & 15.0 & 3.5 & 0.9 & 0.4 & 38.0 & 1.0 & 4.7 & 5.5 & 0.3 & 11.9 & 15.2 \\
\hline BS & 2.4 & 1.5 & 0.0 & 0.0 & 0.0 & 89.9 & 0.3 & 4.4 & 0.7 & 0.2 & 0.7 & 0.0 \\
\hline FR & 9.4 & 22.7 & 7.1 & 1.3 & 0.2 & 18.9 & 0.7 & 4.9 & 8.0 & 0.1 & 2.4 & 24.4 \\
\hline GE & 3.4 & 5.0 & 4.9 & 2.2 & 0.0 & 60.6 & 0.1 & 2.7 & 1.3 & 2.4 & 1.0 & 16.5 \\
\hline GL & 17.8 & 31.0 & 7.1 & 0.6 & 0.4 & 8.9 & 0.7 & 7.8 & 2.9 & 0.0 & 0.3 & 22.4 \\
\hline GR & 16.9 & 36.3 & 5.5 & 0.7 & 0.3 & 10.1 & 0.6 & 6.7 & 3.4 & 0.2 & 0.6 & 18.7 \\
\hline JU & 11.8 & 31.5 & 5.2 & 1.5 & 0.6 & 11.7 & 0.1 & 3.3 & 4.5 & 0.0 & 3.0 & 26.7 \\
\hline LU & 7.3 & 23.0 & 7.7 & 0.5 & 0.1 & 17.9 & 1.1 & 5.2 & 7.4 & 0.0 & 8.6 & 21.3 \\
\hline NE & 15.1 & 23.5 & 6.0 & 3.5 & 0.5 & 23.8 & 0.2 & 2.8 & 0.9 & 0.3 & 1.3 & 22.1 \\
\hline NW & 14.1 & 30.2 & 7.5 & 0.2 & 0.0 & 14.8 & 1.0 & 4.8 & 4.2 & 0.0 & 1.9 & 21.3 \\
\hline OW & 15.9 & 35.6 & 5.6 & 0.7 & 0.4 & 10.7 & 0.0 & 5.7 & 6.1 & 0.0 & 1.8 & 17.3 \\
\hline SG & 9.4 & 24.2 & 7.9 & 1.1 & 0.2 & 20.4 & 0.8 & 6.2 & 5.8 & 0.2 & 6.0 & 17.8 \\
\hline $\mathrm{SH}$ & 5.5 & 10.5 & 6.6 & 3.5 & 0.0 & 44.8 & 2.2 & 4.4 & 3.4 & 2.0 & 2.8 & 14.3 \\
\hline SO & 6.3 & 16.4 & 4.7 & 1.4 & 0.2 & 39.3 & 0.4 & 5.1 & 6.2 & 0.0 & 6.8 & 13.2 \\
\hline SZ & 11.9 & 29.8 & 8.8 & 0.4 & 0.1 & 10.9 & 1.2 & 8.5 & 6.2 & 0.0 & 2.1 & 20.2 \\
\hline TG & 3.0 & 15.1 & 6.1 & 1.3 & 0.2 & 25.0 & 0.5 & 7.5 & 7.6 & 0.2 & 17.4 & 16.2 \\
\hline $\mathrm{TI}$ & 13.0 & 21.1 & 7.7 & 1.5 & 0.1 & 28.5 & 1.1 & 5.7 & 3.0 & 4.8 & 0.0 & 13.5 \\
\hline UR & 12.8 & 33.9 & 10.4 & 0.5 & 0.5 & 11.3 & 1.0 & 8.3 & 3.0 & 0.0 & 0.0 & 18.3 \\
\hline VD & 8.3 & 22.4 & 5.7 & 1.4 & 0.2 & 27.5 & 0.4 & 4.1 & 5.0 & 1.3 & 3.8 & 20.0 \\
\hline VS & 13.4 & 23.4 & 7.3 & 1.9 & 0.3 & 16.2 & 0.2 & 5.3 & 3.5 & 2.0 & 5.3 & 21.3 \\
\hline ZG & 5.4 & 12.0 & 7.1 & 1.6 & 0.1 & 29.7 & 2.7 & 6.9 & 5.6 & 0.0 & 14.3 & 14.5 \\
\hline $\mathrm{ZH}$ & 2.9 & 12.1 & 5.1 & 1.5 & 0.2 & 51.0 & 0.9 & 5.9 & 5.4 & 0.3 & 4.5 & 10.2 \\
\hline $\mathrm{CH}$ & 9.1 & 22.4 & 6.7 & 1.3 & 0.2 & 26.2 & 0.7 & 5.5 & 4.9 & 0.7 & 4.4 & 17.9 \\
\hline
\end{tabular}

Tab 3 Räumliche Verteilung der Bäume ausserhalb des Waldes. Die Zuteilung auf die einzelnen Landnutzungs- beziehungsweise Landbedeckungskategorien basiert auf dem Landschaftsmodell Vector25 der Swisstopo. nähe (49.9\% bzw. 36.3\%). Weil in diesen beiden Kantonen fast alle Bäume ohnehin im Wald stehen (96.2\% bzw. 97.8\%), bedeutet dies, dass praktisch alle vorhandenen Bäume entweder im oder beim Wald anzutreffen sind. In anderen ländlichen Regionen wie Freiburg oder Jura befinden sich Bäume oft in der Nähe von landwirtschaftlich genutzten Flächen. Dies deutet einerseits auf ein Wald-WeideMosaik und andererseits auf eine sukzessive Waldausdehnung auf ehemaligen Weideflächen hin. Beim Kanton Appenzell Ausserrhoden spiegelt sich in der Verteilung der Bäume die Art der Besiedelung wider. Ein überdurchschnittlicher Teil der Bäume liegt im Gebäudeumschwung (15.2\%), annähernd so viele wie in den Siedlungen (17.3\%) In den Kantonen Thurgau, Basel-Landschaft und Zug zeigt sich die landwirtschaftliche Nutzung in einem überdurchschnittlich hohen Obstbaumanteil. In städtischen Regionen wie Genf steht ein Grossteil der Bäume ausserhalb des Waldes im Siedlungsgebiet (60.6\%). Offenbar lassen einerseits der Nutzungsdruck und die Nutzungsform wenig geschlossene Waldfläche zu, andererseits scheint das Bedürfnis nach Grünflächen vor allem in den Städten so gross zu sein, dass ein respektabler Anteil der Siedlungsfläche mit Bäumen bepflanzt wird. Das Siedlungsgebiet spielt also für die Bäume ausserhalb des Waldes und deren Bewirtschaftung ein wichtige Rolle (James et al 2009).

\section{Umgebung von Bäumen}

Waldbäume sind meist umgeben von weiteren Bäumen, zu einem kleineren Teil von Waldlücken, Wiesen oder kleinen Strassen. Die räumliche Zusammensetzung dieser verschiedenen Bodenbedeckungen im Wald ist in Brändli (2010) eingehend beschrieben. Für die Bäume ausserhalb des Waldes ist diese Information erst seit dem LFI3 verfügbar, und sie variiert regional bedeutend stärker. Über die ganze Schweiz gesehen befinden sich die Bäume ausserhalb des Waldes sehr häufig in Waldesnähe (31.5\%; Tabelle 3) oder in urbanen Gebieten (26.2\%). Da das Siedlungsgebiet für die Bäume ausserhalb des Waldes also eine wichtige Rolle spielt, sind der Baumanteil und die Umgebung der Bäume in den zehn grössten Städten genauer analysiert worden (Tabelle 4). Der Baumanteil liegt hier zwischen 15.8\% und 21.7\%. Am meisten Bäume stehen in Schaffhausen und Basel (je 21.7\%), am wenigsten in Thun (15.8\%). Berücksichtigt man auch die Krautund Strauchflächen, beträgt der Grünanteil zwischen 35\% und 51\%. Hier haben nun Luzern (50.9\%) und Winterthur (50.2\%) die Nase vorn. Das Schlusslicht bildet die Stadt Genf mit 38.5\% Grünanteil. Betrachtet man die einzelnen Interpretationsflächen, dann ist der Anteil der Flächen ohne Bäume (17-22\%) und mit dichter Bestockung (3\%-12\%) gering. 


\begin{tabular}{|c|c|c|c|c|c|c|c|c|c|c|}
\hline \multirow[t]{2}{*}{ Stadt } & \multirow{2}{*}{$\begin{array}{l}\text { Sied- } \\
\text { lungs- } \\
\text { fläche } \\
\left(\mathrm{km}^{2}\right)\end{array}$} & \multicolumn{6}{|c|}{$\begin{array}{c}\text { Bodenbedeckung } \\
\text { (\%) }\end{array}$} & \multicolumn{3}{|c|}{$\begin{array}{l}\text { Anteil Inter- } \\
\text { pretationsflächen } \\
(\%)\end{array}$} \\
\hline & & 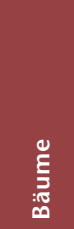 & 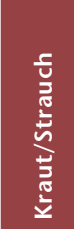 & 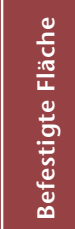 & 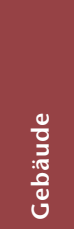 & $\begin{array}{l}\breve{d} \\
\propto\end{array}$ & 气ั & 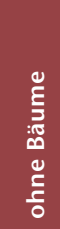 & 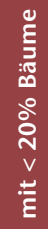 & 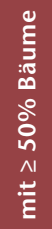 \\
\hline Zürich & 2964 & 16.3 & 28.8 & 27.3 & 27.0 & 0.6 & 100.0 & 17 & 55 & 3 \\
\hline Winterth & 444 & 19.6 & 30.6 & 25.8 & 22.2 & 1.7 & 100.0 & 15 & 45 & 7 \\
\hline Basel & 432 & 21.7 & 14.7 & 33.3 & 29.7 & 0.7 & 100.0 & 21 & 46 & 12 \\
\hline Bern & 416 & 21.0 & 22.3 & 29.0 & 26.2 & 1.6 & 100.0 & 19 & 38 & 8 \\
\hline Lausanne & 287 & 20.1 & 29.6 & 27.4 & 21.8 & 1.0 & 100.0 & 17 & 42 & 8 \\
\hline St. Gallen & 204 & 18.6 & 25.6 & 32.0 & 23.2 & 0.5 & 100.0 & 20 & 40 & 7 \\
\hline Genf & 193 & 19.6 & 18.9 & 28.1 & 33.4 & 0.1 & 100.0 & 22 & 40 & 9 \\
\hline Luzern & 101 & 17.1 & 33.8 & 25.4 & 23.4 & 0.3 & 100.0 & 20 & 53 & 5 \\
\hline Thun & 93 & 15.8 & 27.4 & 27.3 & 28.6 & 1.0 & 100.0 & 19 & 56 & 6 \\
\hline Schaffhausen & 86 & 21.7 & 28.0 & 23.5 & 25.0 & 1.7 & 100.0 & 19 & 33 & 8 \\
\hline
\end{tabular}

Tab 4 Bodenbedeckungsanteile und Anteil an Interpretationsflächen mit definiertem Bestockungsgrad im Siedlungsgebiet der zehn grössten Städte.

\section{Ausblick}

Bäume werden stark mit Wald assoziiert. Die erstmals im Rahmen des LFI3 erhobenen Daten erlauben es nun, ein viel differenzierteres Bild über die Verteilung der Bäume in der Schweiz und in ausgewählten Regionen zu zeichnen. So zeigen die Resultate dieser Untersuchung bereits, dass in einer Mehrheit der Schweizer Kantone der Waldanteil grösser als der Baumanteil ist und dass das Nichtwaldareal bezüglich Bäumen ausgeräumt ist. Als für
Bäume wichtige Gebiete ausserhalb des Waldes stechen die Siedlungen hervor (Abbildung 3).

Die Verteilung der Bäume ist nun schweizweit erfasst. Damit stehen nicht nur die Wald-/Nichtwaldklassen, sondern weitere Merkmale, wie zum Beispiel Baumdeckungsgrad, Mischungsgrad oder Bodenbedeckungsvielfalt, mit welchen Landschaftsgradienten beschrieben werden können, für Auswertungen zur Verfügung (Mathys et al 2006). Aber auch die Art der Nutzung von Bäumen kann nun ohne die bisherige Beschränkung auf das Waldareal untersucht werden. Dies eröffnet neue Möglichkeiten, wie beispielsweise die konsistente Quantifizierung des Holznutzungspotenzials oder weiterer Leistungen der Bäume sowie die Erhebung der mit den verschiedenen Bodenbedeckungselementen assoziierten Nutzungen für die ganze Schweiz.

Die auf dem Luftbild durchgeführte Stichprobenerhebung im LFI3 und im aktuell laufenden LFI4 ist der erste Schritt zu einer umfassenden Erfassung des Zustands und der Veränderung der Bäume in der Schweiz. Für kleine Auswertungseinheiten ist der Stichprobenfehler aber oft so gross, dass insbesondere für Veränderungen keine zuverlässigen Resultate möglich sind. Die vorliegenden Daten können aber als Eichpunkte und zur Qualitätssicherung verwendet werden. Beispielsweise lassen sich anhand dieser Daten mithilfe der Fernerkundung flächendeckende Geodatensätze generieren. Damit kann nicht nur die terrestrische Inventur des LFI optimal vorbereitet, sondern auch die Bodenbedeckung der Schweiz flächendeckend über die entsprechenden Landschaftsgradienten beschrieben werden.

Eingereicht: 16. Dezember 2010, akzeptiert (mit Review): 26. April 2011
Abb 3 Als für Bäume wichtige Gebiete ausserhalb des Waldes stechen die Siedlungen hervor. Foto: Photononstop

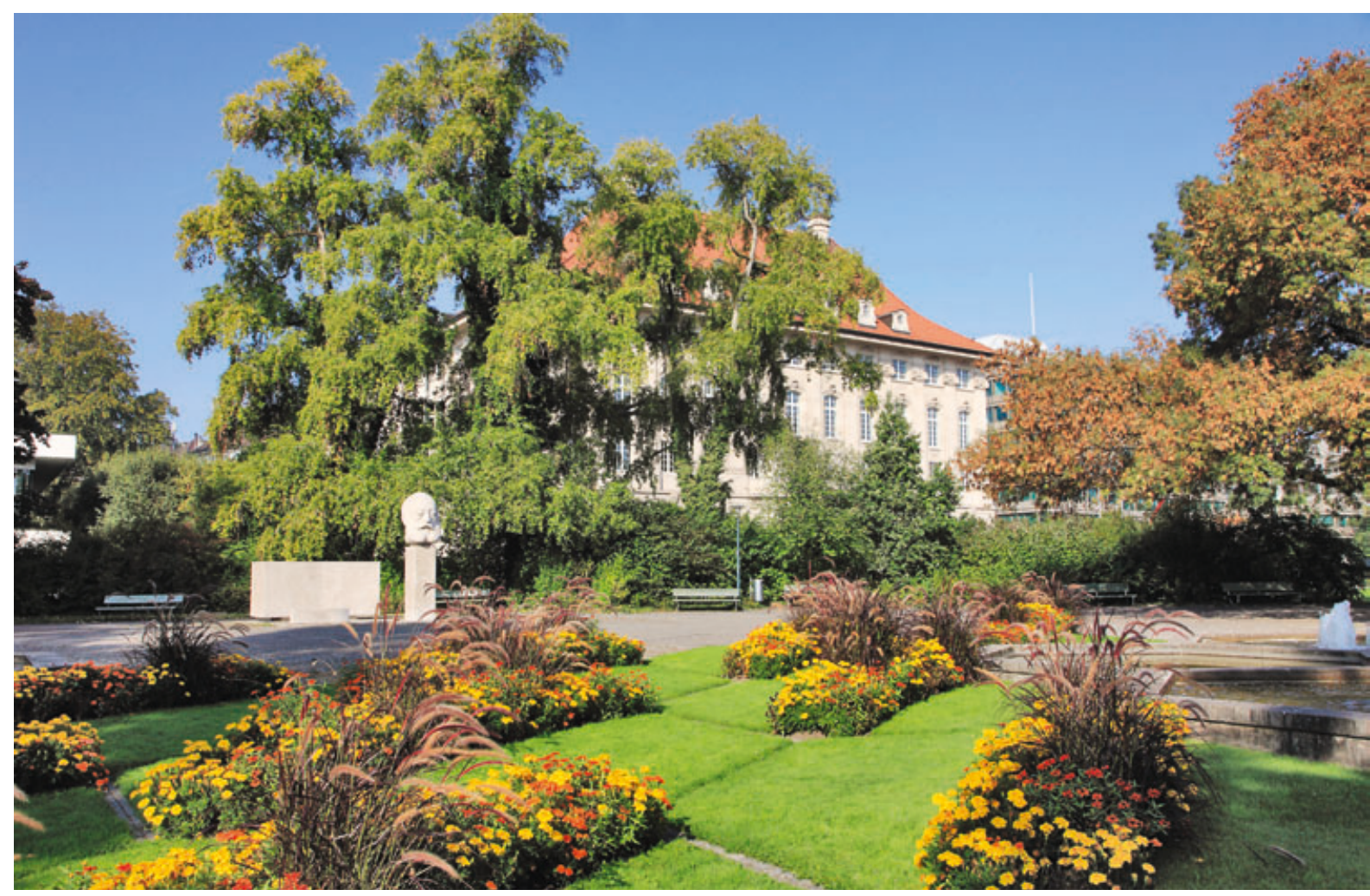




\section{Literatur}

BRÄNDLI UB, EDITOR (2010) Schweizerisches Landesforstinventar: Ergebnisse der dritten Erhebung 2004-2006. Birmensdorf: Eidgenöss Forsch.anstalt Wald Schnee Landschaft. $312 \mathrm{p}$.

BRASSEL P, KÖHL M, SCHNELLBÄCHER HJ, BRÄNDLI UB (1999) Das Landesforstinventar. In: Brassel P, Brändli UB, editors. Schweizerisches Landesforstinventar. Ergebnisse der Zweitaufnahme 1993-1995. Bern: Haupt. pp. 15-25.

BRASSEL P, LISCHKE H, EDITORS (2001) Swiss national forest inventory: Methods and models of the second assessment. Birmensdorf: Swiss Federal Research Institute WSL. 336 p. GINZLER C ET AL (2005) Luftbildinterpretation LFI3. Interpretationsanleitung zum dritten Landesforstinventar. Birmensdorf: Eidgenöss Forsch.anstalt Wald Schnee Landschaft. $85 \mathrm{p}$.

JAMES P ET AL (2009) Towards an integrated understanding of green space in the European built environment. Urban For Urban Gree 8: 65-75.

\section{Die Baumbedeckung in der Schweiz}

Die Anforderungen und die Fragestellung im Zusammenhang mit nationalen Waldinventuren haben sich über die Jahrzehnte geändert. Standen zu Beginn fast ausschliesslich die Holzressourcen im Wald im Vordergrund, so sind heute auch gesellschaftliche und ökologische Funktionen von Interesse. Die Trennung in Wald und Nichtwald bei der Datenerhebung schränkt den Blick auf die Ressource Baum ein. Nicht alle Bäume stehen im Wald, und nicht die ganze Waldfläche ist bestockt. Im dritten Landesforstinventar wurde bei der Luftbildinterpretation die Bodenbedeckung auf einem regelmässigen Stichprobennetz unabhängig von der Nutzung erfasst. So konnten erstmals repräsentative Informationen zur Verteilung der Bäume in der Schweiz gewonnen werden. Die Baumbedeckung der Schweiz beträgt $27.0 \%$, und zwar unabhängig davon, ob die Bäume innerhalb oder ausserhalb des Waldes stehen. Der Waldflächenanteil der Schweiz beläuft sich auf $29.4 \%$ der Landesfläche. Die Waldfläche ist also grösser als die mit Bäumen überschirmte Fläche. Betrachtet man die Lage der Bäume ausserhalb des Waldes, so finden sich diese mehrheitlich entweder in Waldesnähe oder in urbanen Gebieten. Siedlungen sind neben dem Wald der am dichtesten bestockte Raum. Die Daten aus der Luftbildinterpretation des dritten Landesforstinventars erlauben ein differenziertes Bild der Bestockung über die ganze Schweiz. Für kleinräumige Aussagen ist der Stichprobenfehler allerdings gross. Die Daten können aber als Eichpunkte und zur Qualitätssicherung verwendet werden. So lassen sich beispielsweise anhand dieser Daten mithilfe der Fernerkundung flächendeckende Geodatensätze generieren.
KIMMINS JP (2004) Forest ecology: a foundation for sustainable forest management and environmental ethics in forestry. Upper Saddle River: Prentice Hall, 3 ed. 611 p.

LANZ A, GINZLER C (2005) Langfristiges Systemmonitoring wissenschaftliche, technische und organisatorische Herausforderung. Geomatik Schweiz 103: 180-182.

MATHYS L, WILDI O, BRASSEL P (2005) LFI3 - Das Landesforstinventar ist auch ein Landschaftsinventar. Birmensdorf: Eidgenöss Forsch.anstalt Wald Schnee Landschaft, Inf.bl Forsch.bereich Landsch. 64: 1-4.

MATHYS L, GINZLER C, ZIMMERMANN NE, BRASSEL P, WILDI O (2006) Sensitivity assessment on continuous landscape variables to classify a discrete forest area. For Ecol Manage 229: 111-119.

MILLENNIUM ECOSYSTEM ASSESSMENT (2005) Ecosystems and human well-being: Synthesis. Washington D.C.: Island Press. $137 \mathrm{p}$.

\section{La couverture en arbres en Suisse}

Les exigences et la problématique liées aux inventaires forestiers nationaux ont changé au cours des décennies. Alors qu'au début, les ressources en bois de la forêt constituaient l'élément principal, les fonctions sociétales et écologiques revêtent également de l'importance aujourd'hui. La séparation a priori en forêt et non-forêt lors des relevés limite l'interprétation des ressources en arbres. Les arbres ne sont pas tous en forêt, et la surface forestière n'est pas entièrement boisée. Lors de l'interprétation des photos aériennes du troisième Inventaire forestier national suisse, le recouvrement du sol a été étudié indépendamment de l'utilisation des terres sur un réseau d'échantillonnage régulier. C'est la première fois que des informations sur les ressources en arbres sont collectées de façon représentative à l'échelle nationale. En Suisse, la couverture en arbres est de $27.0 \%$, indépendamment du fait que les arbres se trouvent en forêt ou à l'extérieur. Le taux de la couverture en forêts compte $29.4 \%$. L'aire forestière est donc plus grande que la surface couverte par des arbres. Si l'on considère l'emplacement des arbres à l'extérieur de la forêt, ils se trouvent principalement à proximité de la forêt ou dans des zones urbaines. A part la forêt, les zones d'habitation constituent l'aire la plus densément boisée. Les données de I'interprétation des photos aériennes du troisième Inventaire forestier offrent une image nuancée du boisement de toute la Suisse. Toutefois, pour une interprétation à petite échelle, I'erreur d'échantillonnage est considérable. En utilisant l'inventaire existant comme référence et pour la calibration, les méthodes de télédétection promettent de fournir des données géoréférencées généralisées qui comblent cette lacune. 